\title{
Scattering Optimization of Photonic Cluster: From Minimal to Maximal Reflectance
}

\author{
V. V. Prosentsov \\ Stationsstraat 86, 5751 HH Deurne, The Netherlands \\ Correspondence should be addressed to V. V. Prosentsov, prosentsov@yahoo.com
}

Received 30 September 2010; Accepted 19 March 2011

Academic Editor: Dragomir Neshev

Copyright () 2011 V. V. Prosentsov. This is an open access article distributed under the Creative Commons Attribution License, which permits unrestricted use, distribution, and reproduction in any medium, provided the original work is properly cited.

Scattering optimization is a challenging engineering problem with an extensive list of potential applications. For some practical applications, the reflectance of the photonic cluster should be variable from minimal to maximal values. The theoretical investigation of such problem is extremely difficult due to multiple interactions between particles in the cluster. In this paper, the optimization of the light scattered by the photonic cluster made of small particles is studied with the help of the special optimization algorithm and the local perturbation method. It was shown that the photonic cluster can be transformed in such a way that its reflectance will be increased or decreased by several orders of magnitude for selected wavelength and direction.

\section{Introduction}

Electromagnetic wave is known as the fastest carrier of energy and information, and this property makes light indispensable for communications, warfare, and fundamental research. Further still light can be relatively easy supplied into hard-to-reach places via waveguides or fibers [1], and light pattern (its intensity distribution) can be reshaped into desired one by using diffractive optical elements, for example [2].

The diffractive optical element (DOE) usually is a static object with corrugated surface. Light with spatial pattern A interacts with the irregular surface and transforms into pattern $\mathrm{B}$. To change the pattern $\mathrm{A}$ into the pattern $\mathrm{C}$ one needs another DOE or dynamic element inside the DOE. Microelectromechanical systems (MEMSs) are developed for transforming the light with initial pattern A into multiple patterns [3]. In MEMS, mirrors are the dynamical elements, and they are mechanically moved to deflect the light to different directions. Another way of light manipulation is via dynamical change of the refractive index of the medium. It can be done by using nonlinear effects or by moving particles with different refractive index in the medium. The attractive side of the nonlinear effects is that they are always present inside the system, while the drawback is that one needs to use strong electromagnetic fields to show the significance of the effects [4]. The repositioning of the particles (e.g., in the photonic cluster) is much more effective way to locally change the refractive index of the medium, and there are several possible ways to do so. It was demonstrated, for example, that self-assembly and holographic optical trapping allow to create the predefined photonic structures (see the works $[5,6]$ and references therein).

While the materials and the methods for active control of the scatterers are still under development, the theoretical studies have been started already. Recently, the light manipulation was investigated in the work [7] where the weak scattering by the finite object was studied by using the first Born approximation. In practice, the light scattering by photonic cluster may be not weak and it should be studied with other methods. The local perturbation method (LPM) is a suitable tool for such analysis. The LPM correctly describes scattering by small particles of arbitrary shape and with large refractive index (see, e.g., works $[8,9,11]$ and references therein). In the work [10], the LPM was used to study the resonance and zero scattering by the photonic cluster.

In this paper, the light scattered by the cluster made of small particles is optimized by using the special optimization technic and the LPM. By using the optimization, the cluster is transformed in such a way that the scattering from the cluster is significantly minimized or maximized. Several examples 
are presented in the paper, and they show that the scattering by the cluster can be increased or decreased by several orders of magnitude for selected wavelength and direction.

\section{The Formalism}

The formalism used in this section is described in a number of works (see, e.g., $[8,11]$ ), and it will be briefly presented here for convenience and consistency. Consider the photonic cluster positioned at the origin of the coordinates and made of $N$ identical particles whose characteristic size $L$ is small compared to the incident wavelength $\lambda_{0}$. The electric field $\mathbf{E}$ propagating in the host medium filled with the particles is described by the following equation [9]:

$$
\begin{aligned}
(\Delta- & \left.\nabla \otimes \nabla+k_{0}^{2}\right) \mathbf{E}(\mathbf{r}) \\
& +k_{0}^{2} \frac{\left(\varepsilon_{\mathrm{sc}}-\varepsilon_{0}\right)}{\varepsilon_{0}} \sum_{n=0}^{N-1} f\left(\mathbf{r}-\mathbf{r}_{n}\right) \mathbf{E}\left(\mathbf{r}_{n}\right)=\mathbf{S}(\mathbf{r}),
\end{aligned}
$$

where $\mathbf{r}$ and $\mathbf{r}_{n}$ are the radius vectors of the observer and the $n$th particle, respectively, and

$$
k_{0} \equiv \frac{2 \pi}{\lambda_{0}}=\frac{\omega}{c} \sqrt{\varepsilon_{0}}, \quad f\left(\mathbf{r}-\mathbf{r}_{n}\right) \equiv \begin{cases}1, & \text { inside particle } \\ 0, & \text { outside particle }\end{cases}
$$

Here, $\Delta$ and $\nabla$ are the Laplacian and nabla operators, $\otimes$ defines tensor product, $k_{0} \equiv\left|\mathbf{k}_{0}\right|$ is a wave number in the host medium ( $\omega$ is the angular frequency, and $c$ is the light velocity in vacuum), $\varepsilon_{\mathrm{sc}}$ and $\varepsilon_{0}$ are the permittivities of the particles and the medium, respectively, $f$ is the function describing the shape of the scatterers, and $\mathbf{S}$ is the field source. Note that (1) is an approximate one and it is correct when the small scatterers $\left(k_{0} L \ll 1\right)$ are considered.

The solution of (1) can be expressed in the form

$$
\mathrm{E}(\mathbf{r}) \equiv \mathbf{E}_{\mathrm{in}}(\mathbf{r})+\mathbf{E}_{\mathrm{sc}}(\mathbf{r}),
$$

where the scattered field $\mathbf{E}_{\mathrm{sc}}$ is

$$
\begin{gathered}
\mathbf{E}_{\mathrm{sc}}(\mathbf{r})=k_{0}^{2} \frac{\left(\varepsilon_{\mathrm{sc}}-\varepsilon_{0}\right)}{\varepsilon_{0}}\left(\hat{I}+\frac{\nabla \otimes \nabla}{k_{0}^{2}}\right) \sum_{n=0}^{N-1} \mathbf{E}\left(\mathbf{r}_{n}\right) e^{i \mathbf{k}_{0} \cdot \mathbf{r}_{n}} \Phi_{n}(\mathbf{r}) \\
\Phi_{n}(\mathbf{r}) \equiv \int_{-\infty}^{\infty} \frac{\tilde{f}(\mathbf{q}) e^{i \mathbf{q} \cdot\left(\mathbf{r}-\mathbf{r}_{n}\right)}}{\left(q^{2}-k_{0}^{2}\right)} d \mathbf{q} \\
\tilde{f}(\mathbf{q}) \equiv \frac{1}{8 \pi^{3}} \int_{-\infty}^{\infty} f(\mathbf{r}) e^{-i \mathbf{q} \cdot \mathbf{r}} d \mathbf{r}
\end{gathered}
$$

Here, $\hat{I}$ is the $3 \times 3$ unitary tensor in polarization space, and $\mathbf{r}_{n}$ is the radius vector of the $n$th particle. The function $\tilde{f}$ is the Fourier transform of the function $f$. The incident field $\mathbf{E}_{\text {in }}$ is created by the source $\mathbf{S}$ in the host medium (more information can be found in [12]).

Formula (4) is rather a general one and it describes the field scattered by the photonic cluster made of small particles of arbitrary form. The resonance properties and interference between the scatterers are taken into account by the terms $\mathbf{E}\left(\mathbf{r}_{n}\right)$ which should be found by solving the system of $3 N$ linear equations obtained by substituting $\mathbf{r}=\mathbf{r}_{n}$ into (3).

The scattered field (4) can be simplified even further when the distance between the observer and an $n$th scatterer $\left(R_{n}\right)$ is large, that is, when $R_{n} \gg L$. In this case, the integrals (5) can be calculated explicitly, and the scattered field (4) can be presented in the following form:

$$
\mathbf{E}_{\mathrm{sc}}(\mathbf{r})=k_{0}^{2} V \frac{\left(\varepsilon_{\mathrm{sc}}-\varepsilon_{0}\right)}{4 \pi \varepsilon_{0}}\left(\hat{I}+\frac{\nabla \otimes \nabla}{k_{0}^{2}}\right) \sum_{n=0}^{N-1} \mathbf{E}\left(\mathbf{r}_{n}\right) \frac{e^{i k_{0} R_{n}}}{R_{n}},
$$

where

$$
R_{n} \equiv\left|\mathbf{r}-\mathbf{r}_{n}\right| \gg L
$$

Here, $R_{n}$ is the distance between the observation point $\mathbf{r}$ and the radius vector $\mathbf{r}_{n}$ of the $n$th scatterer, and $V$ is the scatterer's volume.

In many practical cases, the distance between the cluster and the observer is much larger than the size of the cluster $\left(|\mathbf{r}| \gg \max \left(\left|\mathbf{r}_{n}\right|\right)\right)$ and the incident wavelength $\left(k_{0}|\mathbf{r}| \gg 1\right)$. In this case, the field (6) can be simplified, and it can be rewritten in the following form:

$$
\mathbf{E}_{\mathrm{sc}}(\mathbf{r}, \lambda)=k_{0}^{2} V \frac{\left(\varepsilon_{\mathrm{sc}}-\varepsilon_{0}\right) e^{i k_{0} r}}{4 \pi \varepsilon_{0} r}(\hat{I}-\mathbf{l} \otimes \mathbf{l}) \sum_{n=0}^{N-1} \mathbf{E}\left(\mathbf{r}_{n}\right) e^{-i k_{0} \mathbf{l} \cdot \mathbf{r}_{n}},
$$

where

$$
\mathbf{l} \equiv \frac{\mathbf{r}}{r}, \quad r \equiv|\mathbf{r}| \gg \max \left(\left|\mathbf{r}_{n}\right|\right), k_{0} r \gg 1 .
$$

The wavelength symbol $\lambda$ is explicitly presented in the notation of the scattered field $\mathbf{E}_{\mathrm{sc}}(\mathbf{r}, \lambda)$ ( that the scattered field depends on the wavelength $\lambda$. It will be used in the following section where the optimization of the cluster reflectance will be described.

\section{Optimization of the Cluster Reflectance}

The idea of my approach is the following. I assume that the scatterers in the cluster can be repositioned in such a way that the intensity of the field scattered by the cluster will be tuned to required value at observation point for selected wavelength. Note that the intensity of the field scattered by the cluster is defined as

$$
I_{\mathrm{sc}}(\mathbf{r}, \lambda) \equiv\left|\mathbf{E}_{\mathrm{sc}}(\mathbf{r}, \lambda)\right|^{2} .
$$

At first, the intensity of the field scattered by the given (not modified) cluster is calculated for the selected wavelength $\lambda_{s}$ at the observation point $\mathbf{r}$. This is the reference intensity. After this, one particle in the cluster is selected, and it is moved a bit (usually for tenth of the particle size) in $\pm x, \pm y$, and $\pm z$ directions. The scattered field is recalculated at $\mathbf{r}$ each time the particle is moved. From the array of the 7 calculated intensities (the reference intensity and 6 others 
due to the moves), the value nearest to the required one is selected, and the particle is placed at the point corresponding to this intensity value. This procedure is performed for all $N$ particles in the cluster and it is repeated several times (several iterations). After several iterations, the particles in the cluster will be rearranged in such a way that the intensity of the scattered light will correspond to the required value at the observation point $\mathbf{r}$ and for the wavelength $\lambda_{s}$.

Several important remarks should be done here. The initial distances between the particles are important. When the distances are too large, the interactions between the scatterers are weak and the optimum may not be found. At short range, there is no space for maneuver. The cubes are better suited for optimization because they have larger volume compared with spheres while the characteristic size (radius) is the same. The move of the scatterers is also important. It can be safe or any (carte blanche). The safe move means that the particles can only move near their initial positions and not further than the half of the period $d / 2$ minus characteristic size $L$. In this case, the scatterers have no chance to collide with each other, that is, the centers of the particles will be separated at least by $2 L$ distance. Note that the number of iterations in the safe mode is limited due to the finite size of the move and that the cluster will preserve its initial shape (plus/minus $d /(2-L)$ ). The carte blanche (any) move means that the particles can be moved into any position and that the number of the iterations is unlimited in principle. The only condition that should be satisfied is that the particles do not collide.

The last note is about the time required for the optimization. During the optimization, the field scattered by the cluster is calculated $7 N$ times during each iteration and this is rather a lengthy process. This is an inherent drawback of the method. The alternative approach will be to solve the set of the following nonlinear equations:

$$
(\widehat{I}-\mathbf{l} \otimes \mathbf{l}) \sum_{n=0}^{N-1} \mathbf{E}\left(\mathbf{r}_{n}\right) e^{-i k_{0} \mathbf{l} \cdot \mathbf{r}_{n}}=\mathbf{h}(\mathbf{r}, \lambda)
$$

where $\mathbf{h}(\mathbf{r}, \lambda)$ is the predefined vector function for maximal or minimal scattering. Equation (11) should be solved in respect to the positions $\mathbf{r}_{n}$ for the set of directions $\mathbf{l}$ or wavelengths $\lambda$. That is even a more difficult and timeconsuming task compared to the optimization proposed here.

By using the optimization described above, the super reflective cluster can be created when the intensity of the scattered field $I_{\mathrm{sc}}(\mathbf{r}, \lambda)$ will be maximized in some directions and minimized in all the other directions. Another important example (closely related to optical cloaking) is the cluster with significantly reduced reflectance for selected wavelength at chosen direction. When the reflectance of the cluster is lower than the sensitivity level of the receiver, this cluster is actually invisible for the observer. The reflectance $\Phi$ of the cluster is defined in the following way:

$$
\Phi \equiv \frac{I_{\mathrm{sc}}(\mathbf{r}, \lambda)}{I_{\mathrm{in}}(0, \lambda)}
$$

where $I_{\text {in }}(0, \lambda)=\left|\mathbf{E}_{\text {in }}(0, \lambda)\right|^{2}$ is the intensity of the incident field at the point $\mathbf{r}=0$.

When the intensity of the field scattered by the cluster is changed in some direction, this effect can be used in directional optical switch. This kind of device can be used for lighthouses, for example, when the spectrum of the field scattered by the cluster is modified in time when the cluster can be used as a dynamic filter.

Below, I present several examples demonstrating the possibility to optimize the cluster reflectance $\Phi$ in chosen direction for the selected wavelength $\lambda_{s}$.

It should be noted that somewhat similar approach was successfully used in the work [13] where the phase of incident field was tuned to maximize the field scattered from the complex object (eggshell and $\mathrm{TiO}$ powder) in chosen direction.

3.1. Examples. Consider the scattering by the spherical photonic cluster made of gold $(A u)$ cubes. The characteristic size of the cubes is $L=10 \mathrm{~nm}$, and they are originally organized (before any optimization) into simple cubic lattice. The clusters with three different periods $\left(d_{1}=2.5 \mathrm{~L}, d_{2}=3.3 \mathrm{~L}\right.$, and $d_{3}=5.0 L$ ) are studied. The radiuses of the given (initial) clusters are set to $\Lambda=3 d$, and the number of the particles in the clusters is $N=123$. The center of the cluster is positioned at the beginning of the coordinates. The incident field $\mathbf{E}_{\text {in }}$ is generated by the point source located at $\mathbf{r}_{s}=\{1,0,0\}$, and the field is described by the formula

$$
\mathbf{E}_{\mathrm{in}}(\mathbf{r}, \lambda)=\mathbf{A} \frac{e^{i k_{0} r_{s}}}{4 \pi r_{s}} e^{-i k_{0}\left(\mathbf{r}_{s} / r_{s}\right) \mathbf{r}},
$$

where the vector $\mathbf{A}=\left\{0, A_{y}, 0\right\}$ has only $y$ component (the incident field is linearly polarized in $y$ direction) and $A_{y}$ is a constant. The scattering by the cluster will be optimized (to minimum and maximum) for the selected wavelength $\lambda_{s}=571 \mathrm{~nm}\left(k_{0} L=0.11\right)$ for the observer located at $\mathbf{r}_{\mathrm{ob}}=\{0.87,0.50,0.00\}$. It is assumed that the permittivity of the host medium is $\varepsilon_{0}=1$ and the permittivity of the scatterers is the same as the permittivity of the bulk $A u$. The actual values of $A u$ permittivity were taken from [14]. Note that the source and the observer are far from the cluster such that $k_{0}\left|\mathbf{r}_{s}\right| \simeq k_{0}\left|\mathbf{r}_{\mathrm{ob}}\right| \gg 1$. The particles in the cluster were rearranged using the carte blanche move in order to maximize or minimize the scattering. The iterations were terminated when the reflectance of the cluster was saturated. For the cluster with the period $d_{1}=2.5 L$, the step move was $\delta_{1}=1.9 \mathrm{~nm}$, and the number of iterations did not exceed 20 . For the clusters with the periods $d_{2}=3.3 \mathrm{~L}$ and $d_{3}=5.0 \mathrm{~L}$, the step move was $\delta_{2}=1.3 \mathrm{~nm}$ and $\delta_{3}=5.9 \mathrm{~nm}$, respectively, and the number of iterations was 20 and 40, respectively.

The examples of the optimization are presented on Figures 1, 2, and 3. The figures show the logarithm (lg) of the reflectance $\Phi$ of the optimized clusters versus the normalized frequency $k_{0} L$. The green and the blue curves show the reflectances of the optimized clusters while, the red curve shows the reflectance of the nonoptimized (given) cluster. The dash-dotted curve shows the reflectance of the single cube with the characteristic size $L$. 


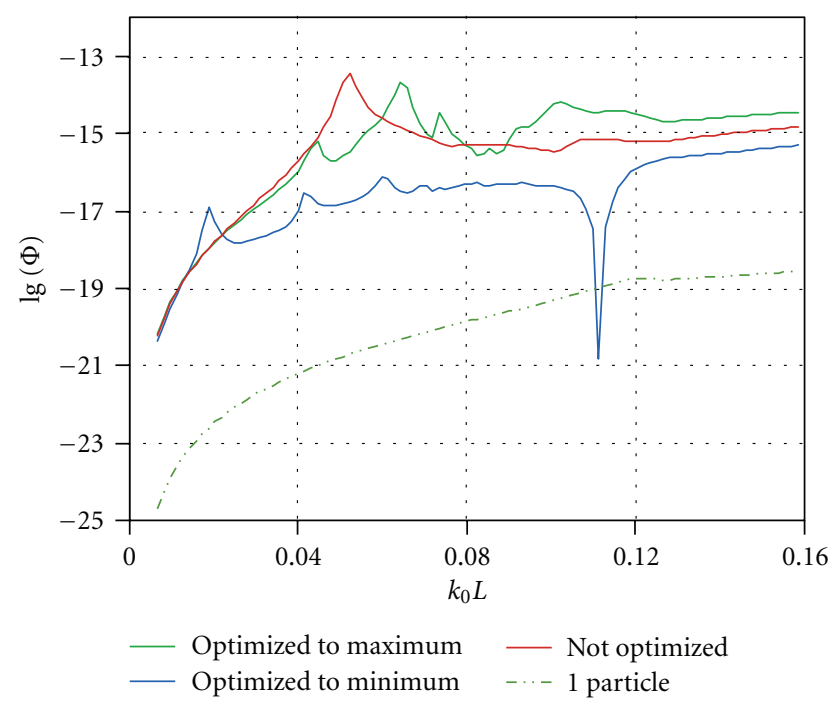

FIgURE 1: The logarithm of the reflectance $\Phi$ of the optimized $A u$ cluster versus the normalized frequency $k_{0} L$. The reflectance was optimized for the frequency $k_{0} L=0.11$ at the observation point $\mathbf{r}_{\mathrm{ob}}=\{0.87,0.5,0.00\}$. The period of the cluster is $d=2.5 \mathrm{~L}$ and $L=10 \mathrm{~nm}$. The green and the blue curves show the reflectance of the cluster optimized for maximal and minimal values, respectively. The red curve shows the reflectance of the nonoptimized cluster, and the green dash-dotted curve shows the reflectance of the single $A u$ cube with the characteristic size $L$.

Consider Figures 1-3 in greater detail. The results of the scattering optimization for the direction $\mathbf{r}=\mathbf{r}_{\mathrm{ob}}$ are presented in these figures. The green curves show the reflectances of the clusters optimized for maximal scattering. The blue curves are the reflectances of the clusters optimized for minimal scattering. It can be seen that the optimization for the minimal reflectance is extremely successful because the minimum is located exactly at the selected wavelength $k_{0} L=0.11$, and the reflectance of the optimized cluster is one or even two orders of magnitude less than the reflectance of the single particle. The optimization for the maximal reflectance is less successful because the maximum is not located at the selected wavelength $k_{0} L=0.11$ (while for the clusters of Figures 2 and 3, the maximum is very distinct and it is located at $\left.k_{0} L=0.107\right)$. Note also that the reflectance of the optimized cluster is only one order of magnitude more than the reflectance of the original cluster.

Examination of Figures 1-3 reveals two important things. The first important thing is that the optimization works because the reflectance of the optimized cluster has clear minimum or maximum for the chosen wavelength (or very near to it) and the direction. The second important thing is that the reflectance has deep or peak not only for designed wavelength but also for other wavelengths (and directions). It should be emphasized that the scattering was optimized for one direction and for one wavelength only, and that is why the optimization is effective at least for narrow spectrum of wavelengths (and scattering directions).

It is worthwhile to know how the particles are redistributed in the optimized cluster. For this purpose, Figure 4

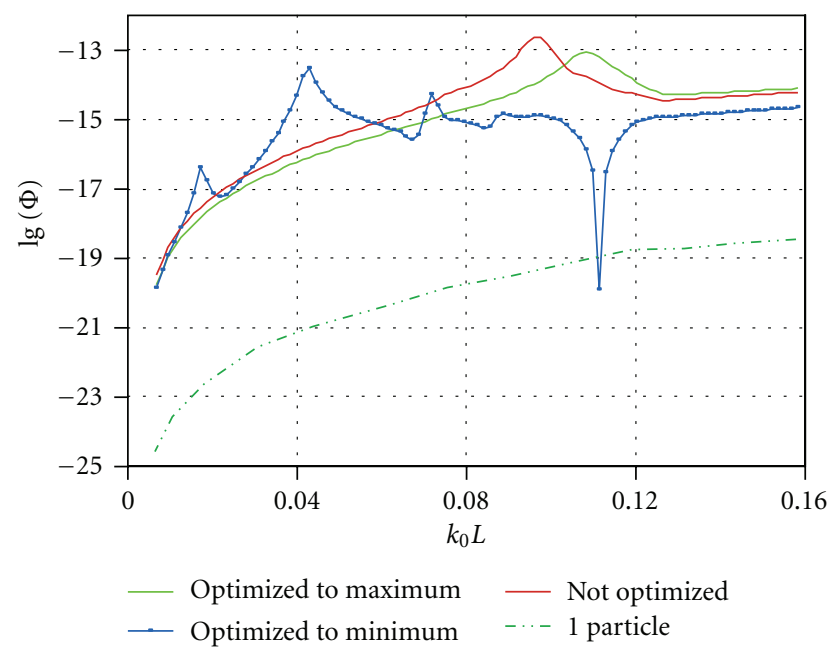

Figure 2: The logarithm of the reflectance $\Phi$ of the optimized $A u$ cluster versus the normalized frequency $k_{0} L$. The reflectance was optimized for the frequency $k_{0} L=0.11$ at the observation point $\mathbf{r}_{\mathrm{ob}}=\{0.87,0.5,0.00\}$. The period of the cluster is $d=3.3 \mathrm{~L}$ and $L=10 \mathrm{~nm}$. The green and the blue curves show the reflectance of the cluster optimized for maximal and minimal values, respectively. The red curve shows the reflectance of the nonoptimized cluster, and the green dash-dotted curve shows the reflectance of the single $A u$ cube with the characteristic size $L$.

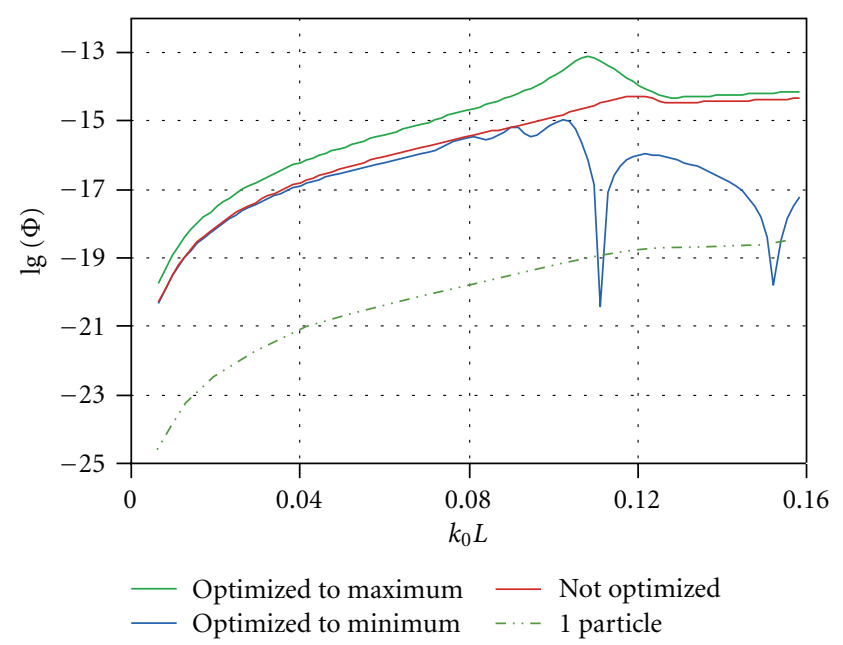

Figure 3: The logarithm of the reflectance $\Phi$ of the optimized $A u$ cluster versus the normalized frequency $k_{0} L$. The reflectance was optimized for the frequency $k_{0} L=0.11$ at the observation point $\mathbf{r}_{\mathrm{ob}}=\{0.87,0.5,0.00\}$. The period of the cluster is $d=5 \mathrm{~L}$ and $L=10 \mathrm{~nm}$. The green and the blue curves show the reflectance of the cluster optimized for maximal and minimal values, respectively. The red curve shows the reflectance of the nonoptimized cluster, and the green dash-dotted curve shows the reflectance of the single $A u$ cube with the characteristic size $L$.

is presented where the positions of the scatterers are shown in the initial (not optimized) cluster and in the cluster optimized for the minimal scattering. The figure shows that the optimized cluster consists mainly of two parts which 


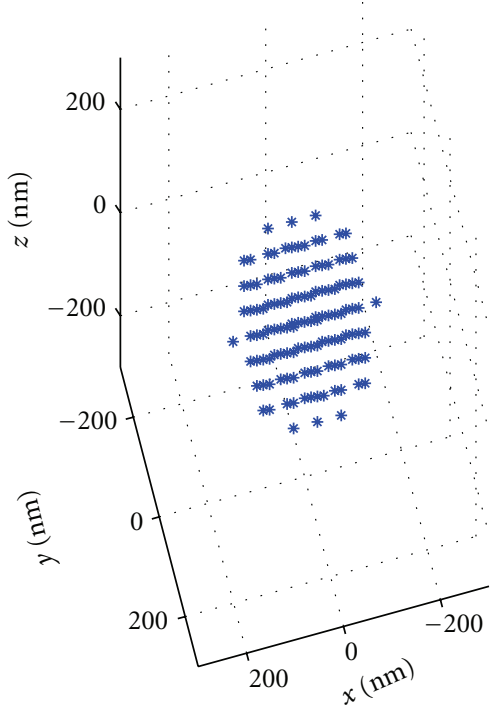

(a)

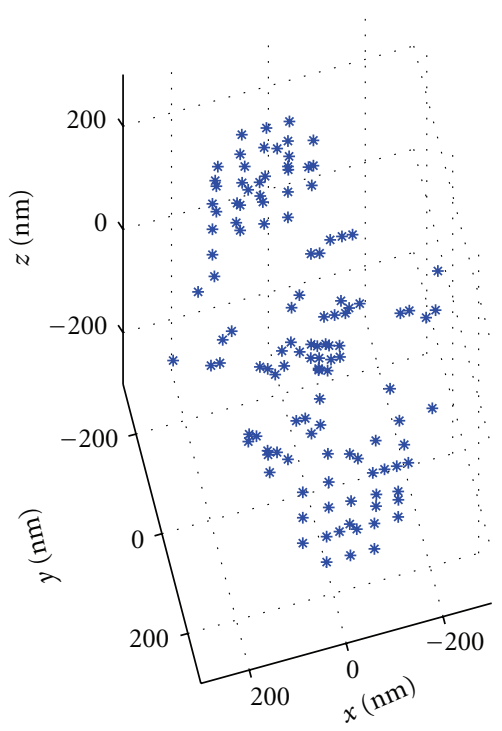

(b)

FIGURE 4: The positions of the scatterers in the initial (not optimized) cluster and in the cluster optimized for the minimal scattering. The reflectance was optimized for the normalized frequency $k_{0} L=0.11$ at the observation point $\mathbf{r}_{\mathrm{ob}}=\{0.87,0.5,0.00\}$. The period of the initial cluster is $d=5 L$ and $L=10 \mathrm{~nm}$.

probably interact in a destructive way, and as the result, the total scattering is extremely weak.

\section{Conclusions}

The special optimization technic has been developed for the optimization of the light scattering by the photonic cluster made of small particles. The reflectance of the photonic cluster has been optimized to maximal and minimal values for selected wavelength and direction by using the proposed optimization algorithm.

\section{Acknowledgments}

The author would like to express his gratitude to Professor V. Freilikher for critical comments and helpful discussions. He would like to thank also I. Nikolaev for his comments and important suggestions. He thanks his wife Lucy for understanding and spiritual support.

\section{References}

[1] M. Bass, Ed., Handbook of Optics: Fiber Optics and Nonlinear Optics, vol. 4, chapter 1, McGraw-Hill, New York, NY, USA, 2nd edition, 2001.

[2] D. C. O'Shea et al., Diffractive Optics: Design, Fabrication, and Test, SPIE Tutorial Texts in Optical Engineering, TT62, SPIE Press, Washington, DC, USA, 2004.

[3] A. Liu, Photonic MEMS Devices: Design, Fabrication and Control, CRC Press, Boca Raton, Fla, USA, 2009.

[4] R. W. Boyd, Nonlinear Optics, Academic Press, New York, NY, USA, 3rd edition, 2008.
[5] M. Humar and I. Muševič, "3D microlasers from selfassembled cholesteric liquid-crystal microdroplets," Optics Express, vol. 18, no. 26, pp. 26995-27003, 2010.

[6] Y. Roichman and D. G. Grier, "Holographic assembly of quasicrystalline photonic heterostructures," Optics Express, vol. 13, no. 14, pp. 5434-5439, 2005.

[7] T. D. Gerke and R. Piestun, "Aperiodic volume optics," Nature Photonics, vol. 4, no. 3, pp. 188-193, 2010.

[8] P. C. Chaumet, A. Rahmani, and G. W. Bryant, "Generalization of the coupled dipole method to periodic structures," Physical Review B, vol. 67, no. 16, Article ID 165404, 5 pages, 2003.

[9] F. G. Bass, V. D. Freilikher, and V. V. Prosentsov, "Small nonlinear particles in waveguides and resonators," Journal of Electromagnetic Waves and Applications, vol. 14, no. 12, pp. 1723-1741, 2000.

[10] V. Prosentsov, "Resonance and zero scattering of light by spherical photonic clusters: analytical approach," Optical Engineering, vol. 49, no. 12, Article ID 128001, 6 pages, 2010.

[11] B. T. Draine and P. J. Flatau, "Diserete-dipole approximation for periodic targets: theory and tests," Journal of the Optical Society of America A, vol. 25, no. 11, pp. 2693-2703, 2008.

[12] J. D. Jackson, Classical Electrodynamics, chapter 6, John Wiley and Sons, New York, NY, USA, 3rd edition, 1998.

[13] I. M. Vellekoop and A. P. Mosk, "Phase control algorithms for focusing light through turbid media," Optics Communications, vol. 281, no. 11, pp. 3071-3080, 2008.

[14] E. D. Palik, Handbook of Optical Constants of Solids, vol. 2, Academic Press, New York, NY, USA, 1991. 

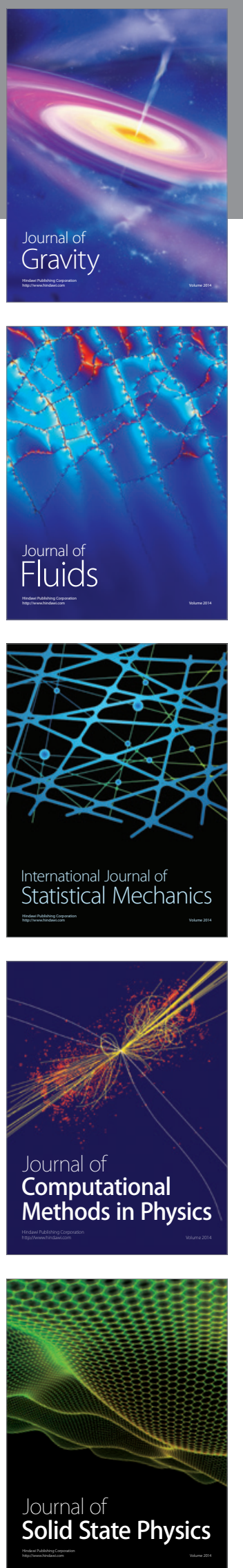

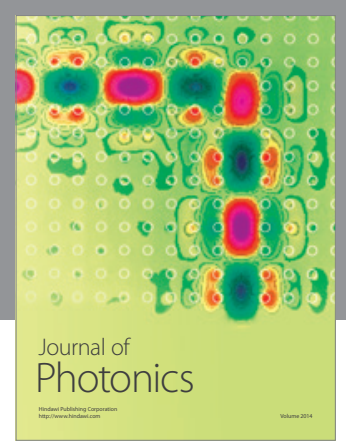

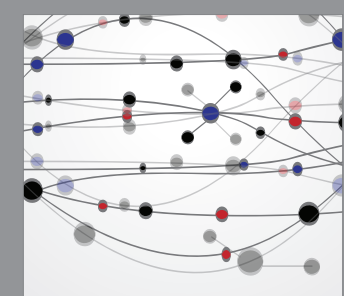

The Scientific World Journal
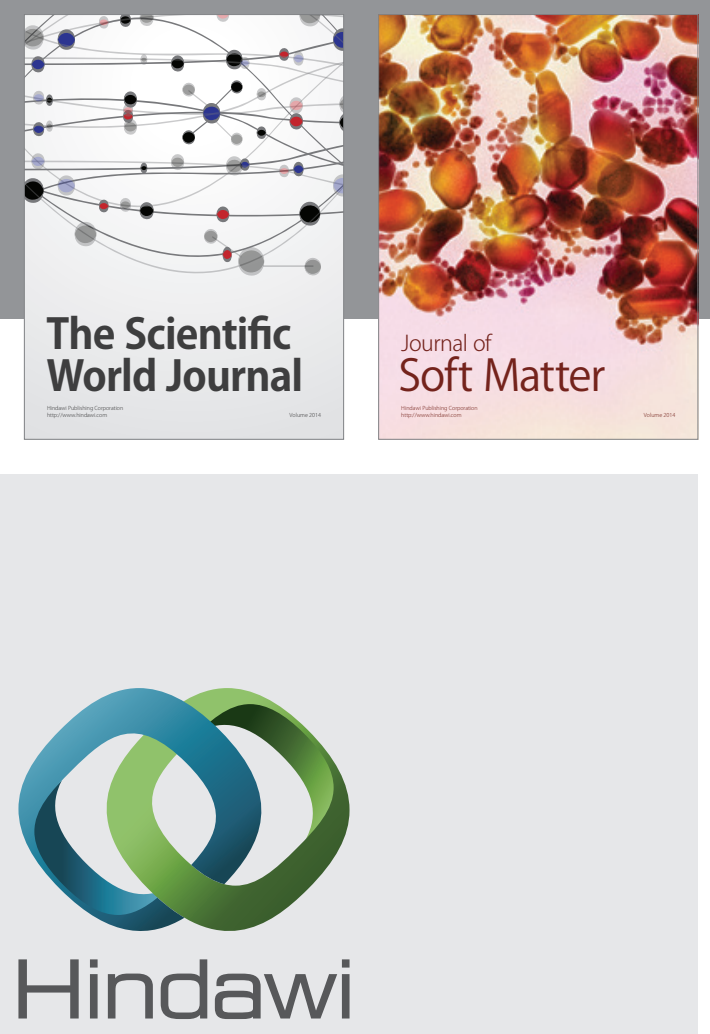

Submit your manuscripts at

http://www.hindawi.com
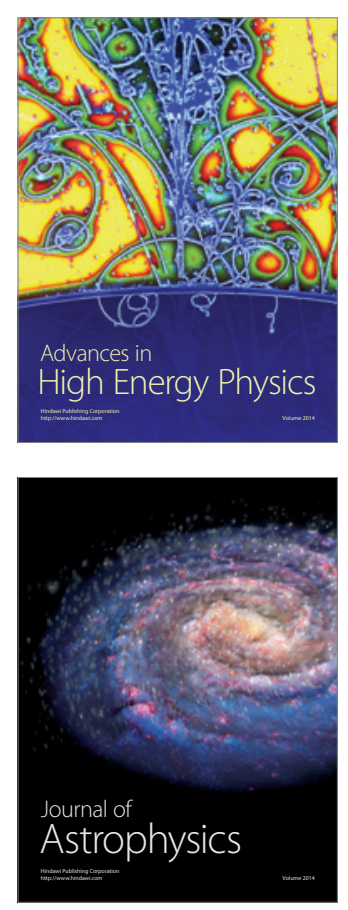
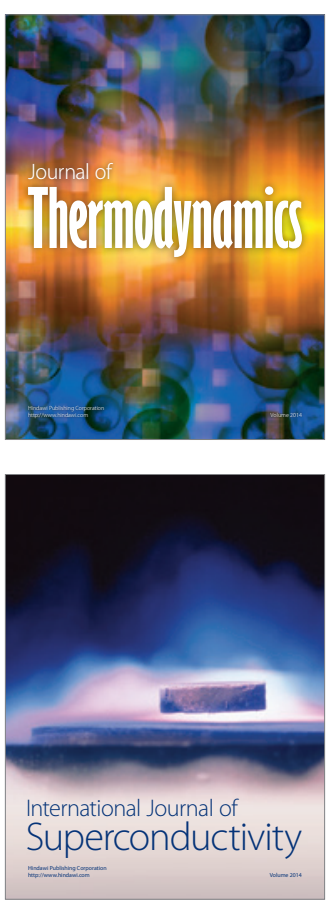
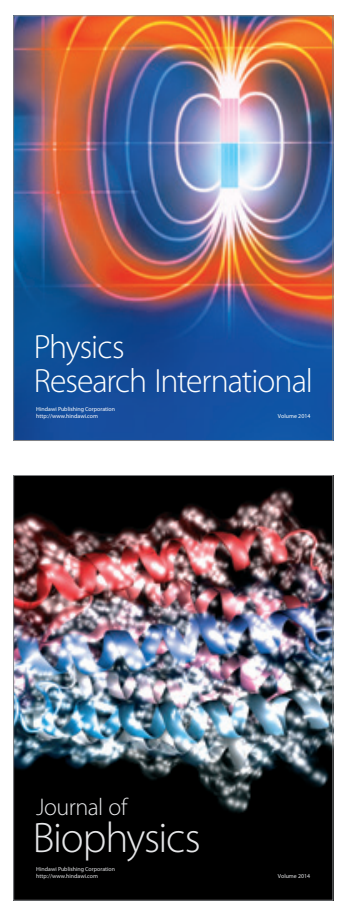
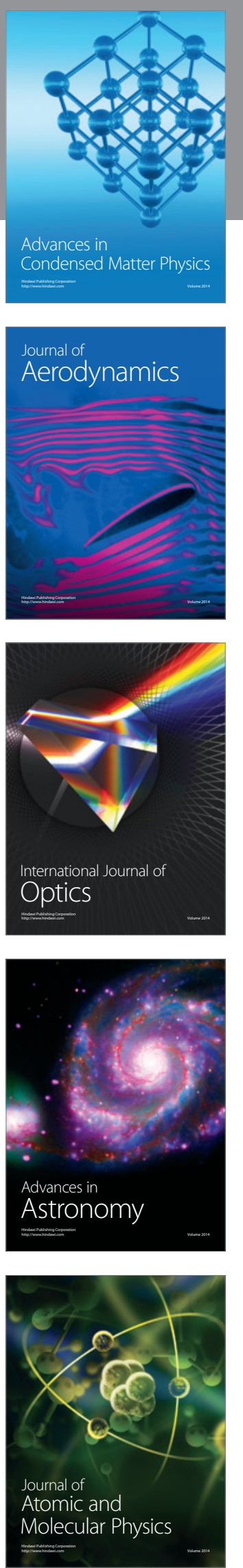